\title{
A test of the extreme value type I assumption in the bus engine replacement model
}

\author{
Larsen, Bradley J ; Oswald, Florian ; Reich, Gregor ; Wunderli, Dan
}

\begin{abstract}
This note tests the assumption of dynamic discrete choice models that underlying utility shocks have an extreme value type I distribution. We find that extreme value type I shocks cannot be rejected in most specifications of the Rust (1987) bus engine replacement model.
\end{abstract}

DOI: https://doi.org/10.1016/j.econlet.2012.02.031

Posted at the Zurich Open Repository and Archive, University of Zurich ZORA URL: https://doi.org/10.5167/uzh-61679

Journal Article

Originally published at:

Larsen, Bradley J; Oswald, Florian; Reich, Gregor; Wunderli, Dan (2012). A test of the extreme value type I assumption in the bus engine replacement model. Economics Letters, 116(2):213-216.

DOI: https://doi.org/10.1016/j.econlet.2012.02.031 


\title{
A Test of the Extreme Value Type I Assumption in the Bus Engine Replacement Model
}

\author{
Bradley J. Larsen, Florian Oswald, Gregor Reich, Dan Wunderli ${ }^{\mathrm{d}}$
}

February 22, 2012

\begin{abstract}
This note tests the assumption of dynamic discrete choice models that underlying utility shocks are distributed extreme value type I. We find that extreme value type I shocks cannot be rejected in most specifications of the Rust (1987) bus engine replacement model.
\end{abstract}

Keywords: Dynamic discrete choice; Extreme value type I. JEL Classification: C25, C61.

\footnotetext{
${ }^{a}$ Corresponding author; Department of Economics, Massachusetts Institute of Technology, 50 Memorial Dr. E52-391 Cambridge, MA 02142. Email: blarsen@mit.edu; Tel: (617) 2862714; Fax: (617) 253-1330.

${ }^{\mathrm{b}}$ Department of Economics, University College London, Gower Street, London, WC1E 6BT. Email: f.oswald@ucl.ac.uk

${ }^{\mathrm{c}}$ Department of Business Administration, University of Zurich, Moussonstrasse 15, 8044 Zurich. Email: gregor.reich@uzh.ch

${ }^{\mathrm{d}}$ Department of Economics, University of Zurich, Wilfriedstrasse 6, 8032 Zurich. Email: dan.wunderli@econ.uzh.ch
} 


\section{Introduction}

In dynamic discrete choice models, by far the most common distributional assumption for the unobserved, random component of utility is that it is drawn from the extreme value type I distribution (see the recent survey by Aguirregabiria and Mira (2010)). This assumption is not based on theory, but rather on computational convenience, and perhaps justifiably so: dynamic discrete choice models can be difficult to estimate, and the extreme value assumption reduces an important dimension of complexity by yielding closed-form solutions for choice probabilities as well as for value functions.

In spite of its prevalence, the extreme value assumption of discrete choice models has rarely been tested. It is our belief that it is important to test the sensitivity of econometric models to its assumptions, whether these assumptions are made for theoretical or computational reasons. This paper contributes to the literature by testing the extreme value assumption in the now well-known engine-replacement model of Rust (1987), a pioneering paper in dynamic discrete choice.

It is important to note that McFadden and Train (2000) show that any random utility model, such as in static demand estimation, can be approximated arbitrarily closely using the extreme value type I distribution for the idiosyncratic component of utility as long as the model includes a sufficiently flexible mixing distribution of random coefficients. With the Rust (1987) model, however, as with many other dynamic discrete choice settings, there are no random coefficients and the only source of uncertainty is the extreme value type I error, and hence the extreme value assumption may potentially be restrictive.

With the original data from Rust's paper, we use contrained optimization and numerical quadrature rules to test the extreme value distribution against several flexible distributions of the exponentiated generalized beta family, which nest the extreme value distribution as a special case. We find, surprisingly, that in most cases, the hypothesis of extreme value errors cannot be rejected, suggesting that, in Rust's data and model, the extreme value assumption, while useful from a computational standpoint, may also be a reasonable modeling assumption. When the assumption can be rejected at marginally significant levels, we demonstrate the implications which our adaptive estimation has for the model.

\section{The Bus Engine Replacement Model}

In the engine-replacement model of Rust (1987), a single agent decides each period whether or not to replace a bus engine. The agent's per-period utility is

$$
u\left(x_{t}, i, \theta\right)+\varepsilon_{t}(i)= \begin{cases}-R C-c\left(0, \theta_{1}\right)+\varepsilon_{t}(1) & \text { if } i=1, \\ -c\left(x_{t}, \theta_{1}\right)+\varepsilon_{t}(0) & \text { if } i=0,\end{cases}
$$

where $i=1$ represents the agent's decision to replace the engine and $i=0$ the decision to not replace. $x_{t}$ is the engine's mileage at time $t$, discretized into 90 
mileage bins, and $\varepsilon_{t}(i)$ is a choice-specific shock to utility (observable to the agent but not to the econometrician). $R C$ (replacement cost) and $\theta_{1}$ (regular maintenance cost) are two of the parameters to be estimated. The function $c\left(x, \theta_{1}\right)$ represents the costs of maintenance on an engine with current mileage $x$. As in the main specification of Rust (1987), we take $c\left(x, \theta_{1}\right)=0.001 \theta_{1} x$.

When the agent discounts future utility with discount factor $\beta$, the agent's value function can be written as

$$
V_{\theta}\left(x_{t}, \varepsilon_{t}\right)=\max _{i \in\{0,1\}} u\left(x_{t}, i, \theta\right)+\varepsilon_{t}(i)+\beta E\left[V_{\theta}\left(x_{t+1}, \varepsilon_{t+1}\right) \mid x_{t}, i\right]
$$

where the subscript $\theta$ is used to denote the dependence of the value function on the full parameter vector, $\theta$, which includes $R C$ and $\theta_{1}$, as well as $\theta_{2}$, which represents any parameters of the unobserved error distribution, and $\theta_{3}$, which represents the parameters of the mileage transition probability.

The expected continuation value can be written as

$$
E\left[V_{\theta}\left(x_{t+1}, \varepsilon_{t+1}\right) \mid x_{t}, i\right]=\int_{x_{t+1}} \int_{\mathcal{E}_{t+1}} V_{\theta}\left(x_{t+1}, \varepsilon_{t+1}\right) P\left(d x_{t+1}, d \varepsilon_{t+1} \mid x_{t}, i, \theta\right)
$$

where

$$
P\left(x_{t+1}, \varepsilon_{t+1} \mid x_{t}, i, \theta\right)=q\left(\varepsilon_{t+1} \mid \theta_{2}\right) p\left(x_{t+1} \mid x_{t}, i, \theta_{3}\right)
$$

with the mileage transition probability defined in $\theta_{3}$, a three-element vector given by

$$
p\left(x_{t+1} \mid x_{t}, i, \theta_{3}\right)= \begin{cases}\theta_{30} & \text { if } x_{t+1}=x_{t} \\ \theta_{31} & \text { if } x_{t+1}=x_{t}+1 \\ \theta_{32} & \text { if } x_{t+1}=x_{t}+2\end{cases}
$$

Now let $m_{i t} \equiv u\left(x_{t}, i, \theta\right)+\beta E\left[V_{\theta}\left(x_{t+1}, \varepsilon_{t+1}\right) \mid x_{t}, i\right]$. Also, let $\eta_{t}=\varepsilon_{t}(1)-$ $\varepsilon_{t}(0)$. Then, under the assumption that $\varepsilon_{t+1}$ is distributed extreme value type I, $\eta_{t}$ will be distributed logistic. Thus the probability that the agent chooses to not replace the engine in mileage state $x_{t}$ is given by the familiar logit formula

$$
\begin{aligned}
\operatorname{Pr}\left(i_{t}=0 \mid x_{t}, \theta\right) & =\frac{1}{1+e^{-\left(m_{0 t}-m_{1 t}\right)}} \\
& =F\left(m_{0 t}-m_{1 t}, \theta_{2}\right)
\end{aligned}
$$

$F\left(\cdot, \theta_{2}\right)$ denotes the logistic distribution, with scale and location parameters normalized, and hence $\theta_{2}$ is empty. Below we will discuss more general distributions which do include parameters contained in $\theta_{2}$.

Also note that

$$
\begin{aligned}
\int_{\mathcal{E}_{t}} V_{\theta}\left(x_{t}, \varepsilon_{t}\right) q\left(\varepsilon_{t}, \theta_{2}\right) d \varepsilon_{t}= & m_{0 t} F\left(m_{0 t}-m_{1 t}, \theta_{2}\right)+m_{1 t}\left(1-F\left(m_{0 t}-m_{1 t}, \theta_{2}\right)\right) \\
& +E\left[\eta_{t} \mid \eta_{t}>m_{0 t}-m_{1 t}\right]\left(1-F\left(m_{0 t}-m_{1 t}, \theta_{2}\right)\right) \\
= & \ln \left(e^{m_{0 t}}+e^{m_{1 t}}\right)
\end{aligned}
$$


where the final line follows from the closed-form solution of the maximum of extreme value type I distributed random variables, familiar from the discrete choice literature.

The likelihood function of an observed sequence of mileage states and decisions, $\left(\left\{x_{t}\right\}_{t=1}^{T},\left\{i_{t}\right\}_{t=1}^{T}\right)$, for a particular bus is given by

$$
L\left(\left\{x_{t}\right\}_{t=1}^{T},\left\{i_{t}\right\}_{t=1}^{T} \mid x_{0}, i_{0}, \theta\right)=\prod_{t=1}^{T} P\left(i_{t} \mid x_{t}, \theta\right) p\left(x_{t} \mid x_{t-1}, i_{t-1}, \theta_{3}\right)
$$

which can be maximized using the constrained maximization procedure described below.

\section{Estimation}

We solve for the model's parameters following the "Mathematical Program with Equilibrium Constraints" (MPEC) approach proposed by Judd and Su (ming). That is, we maximize the likelihood in (3) subject to (1) holding at each state $x$. As in Rust (1987), we use a discount factor of $\beta=0.9999$.

To test the assumption that $\left(\varepsilon_{t}(0), \varepsilon_{t}(1)\right)$ are distributed extreme value type I, we choose distributions of $\eta_{t}$ which nest the logistic distribution. Specifically, we choose distributions from the exponentiated generalized beta of the second kind (EGB2) family. The EGB2 has the following CDF and density:

$$
\begin{aligned}
& F_{E G B 2}(\eta ; p, q)=\mathcal{B}\left(\frac{1}{1+e^{\eta+\psi(p)-\psi(q)}} ; p, q\right) \\
& f_{E G B 2}(\eta ; p, q)=\frac{e^{p(\eta+\psi(p)-\psi(q))}}{B(p, q)\left(1+e^{\eta+\psi(p)-\psi(q)}\right)^{p+q}}
\end{aligned}
$$

where $p$ and $q$ are distributional parameters determining the shape (skewness and kurtosis) of the distribution, $\psi$ is the digamma function (the first derivative of the $\log$ of the gamma function), $B(p, q)$ is the beta function. Finally, $\mathcal{B}(\cdot ; p, q)$ is the incomplete beta function.

Through the parameters $p$ and $q$, the EGB2 distribution allows for a wide range of standardized skewness $([-2,2])$ and standardized kurtosis $([3,9])$, as shown in Hansen et al. (2007). The logistic distribution is a special case of the EGB2, with $p=q=1$, and allows for zero skewness and only one level of kurtosis (a value of 4.2). Formulas for the skewness and kurtosis of the EBG2 are found in McDonald and $\mathrm{Xu}$ (1995).

Two special cases of the EGB2 which nest the logistic distribution while still being flexible are the exponentiated Burr type 3 distribution (EBurr3), given by $F_{E G B 2}(\cdot ; p=1, q)$ and the exponentiated Burr type 12 distribution (EBurr12), given by $F_{E G B 2}(\cdot ; p, q=1)$. These latter two distributions have convenient closed form CDFs found in Hansen and McDonald (2002).

However, unlike with the logistic disribution, the truncated expectation, $E\left[\eta_{t} \mid \eta_{t}>m_{0 t}-m_{1 t}\right]$, found in (2), does not have a closed form solution under 
these more flexible distributions. We evaluate this object using Gauss-Laguerre quadrature (see Kythe and Schäferkotter (2005) for details). As demonstrated below, we find this method to be highly accurate.

\section{Results}

We use the original data from Rust (1987). This data consists of many buses, each with monthly data on mileage readings as well as the agent's decision to replace the engine or not. The buses are divided into four groups based on their characteristics. See Rust (1987) for more details on the data.

We first replicate the Rust (1987) results using the closed-form which arises from the extreme value type I assumption. We then performed the same estimation but with Gauss-Laguerre quadrature, using 100 Laguerre nodes, rather than using the closed-form solution. The results of these replication exercises for bus groups 1-3 are displayed in Table 1 in the columns labeled "Closed Form" and "Gauss-Laguerre." The results from Rust (1987), Table IX, are also displayed for comparison. ${ }^{1}$ As can be seen, the numerical quadrature results agree with the closed-form solution, and our results replicate Rust's quite closely.

To test the extreme value assumption, we estimate the model using each distribution described above and perform a likelihood ratio test. Table 2 shows the results, with a separate panel for the sample containing bus groups 1-3, bus group 4 alone, and bus groups 1-4. Note that the mileage transition parameters, $\theta_{30}$ and $\theta_{31}$, are omitted from Table 2 because they do not depend on the distributional assumption of $\eta_{t}$.

In Table 2, note that the parameter estimates vary slightly depending on the distributional assumption used. For example, in each panel, the replacement cost $(\mathrm{RC})$ is nearly the same across distributions. The regular maintenance cost, $\theta_{1}$, on the other hand, is approximately twice as large under the logistic assumption than it is under the Eburr12. However, in each case a Hausman test fails to reject when comparing $\theta_{1}$ and $R C$ under the logistic to each of the flexible distributions, as shown in the final column of each panel.

Observe that the flexible distributions pick up some positive skewness. In other words, the distribution of the unobserved component of utility appears to be skewed to the right, or have a long right tail, having relatively few high values and having more mass concentrated at the left of the distribution. Recalling that this is the distribution of $\eta_{t}=\varepsilon_{t}(1)-\varepsilon_{t}(0)$, this implies that large shocks to utility favoring engine replacement are less likely to occur than would be predicted by assumption that $\left(\varepsilon_{t}(0), \varepsilon_{t}(1)\right)$ is distributed extreme value type I. Table 2 displays mild differences in kurtosis as well.

Observe, however, that the p-value from the likelihood ratio (LR) test would not allow one to reject the extreme value type I (or here, the logistic) assumption at conventional $(5 \%)$ levels. This suggests that the extreme value assumption may be appropriate in this data and model.

\footnotetext{
${ }^{1}$ Rust (1987) standard errors come from the outer product of the gradient. All other standard errors are bootstrapped at the bus level.
} 
Only in the final panel of Table 2, bus groups 1-4, when comparing the logistic and EBurr3 distributions, does the LR test yield a marginally significant p-value of 0.0725 . If the hypothesis of extreme value errors could be rejected, it would be interesting to identify the effects of this distributional assumption on the model. For this purpose, we examine more closely the estimated conditional choice probabilities for bus groups 1-4 under the logistic distribution and under the EBurr3, shown in Figure 1.

Observe in Figure 1, panel (a), that at low mileage states, the two distributions predict similar absolute probabilities of engine replacement. At high mileage states (which are not reached as often in the data), the logistic predicts a much higher likelihood of engine replacement than does the EBurr3. This result may arise because engine replacement rarely occurs, and, being a tail occurrence, is predicted differently by the flexible EBurr3 than by the logistic. The plot of the relative probabilities in panel (b) demonstrates that at some mid-level mileage states, the EBurr3 does predict a slightly higher replacement probability. Panel (b) also reveals that at many mileage states, particularly low and high mileage states, the $95 \%$ confidence interval of the relative probabilies (marked by the dashed lines) does not contain unity, meaning the choice probabilities differ significantly from one another.

\section{Conclusion}

This paper tests an assumption which is extremely common in dynamic (and static) discrete choice estimation: that the unobserved component of utility is distributed according to an extreme value type I distribution. This assumption is not made for theoretical reasons but rather because it yields closed-form solutions for choice probabilities and for expected continuation values. If the assumption is harmless, its usefulness makes it an excellent modeling choice. If, however, the model is quite sensitive to the distribution of unobserved shocks, researchers should be aware of this and consider these sensitivities.

Although the results of this paper are only for one particular example, the procedure could be followed for other dynamic binary choice models. In particular, we demonstrate that without the closed-form solutions provided by the extreme value type I distribution, Gauss-Laguerre integration can be easily and accurately performed. Also, this paper only considers a dynamic binary choice model, but similar approaches using numerical cubature rules could be applied to dynamic choice models with multiple discrete choices.

\section{Acknowledgements}

We would like to thank Ken Judd, Whitney Newey, and Karl Schmedders, as well as participants at the Chicago-Argonne Institute for Computational Economics 2010, for helpful comments and advice. 


\section{References}

Aguirregabiria, V. and Mira, P. (2010). Dynamic discrete choice structural models: A survey. Journal of Econometrics, 156:38 - 67.

Hansen, C. B., McDonald, J. B., and Theodossiou, P. (2007). Some flexible parametric models for partially adaptive estimators of econometric models. economics (the open access, open assessment e-journal), 7 .

Hansen, J. V. and McDonald, J. B. (2002). A generalized model for predictive data mining. Information Systems Frontiers, 4(2):179-186.

Judd, K. L. and Su, C.-L. (forthcoming). Constrained optimization approaches to estimation of structural models. Econometrica.

Kythe, P. and Schäferkotter, M. (2005). Handbook of computational methods for integration, volume 1. Chapman \& Hall/CRC.

McDonald, J. B. and Xu, Y. J. (1995). A generalization of the beta distribution with applications. Journal of Econometrics, 66:133-152.

McFadden, D. and Train, K. (2000). Mixed MNL models for discrete response. Journal of Applied Econometrics, 15(5):447 - 470.

Rust, J. (1987). Optimal replacement of GMC bus engines: An empirical model of Harold Zurcher. Econometrica, 55(5):999 - 1033. 


\begin{tabular}{cccc}
\hline \hline & \multicolumn{3}{c}{ Bus Groups 1,2,3 $(N=3,864)$} \\
\cline { 2 - 4 } & Rust 1987 & Closed Form & Gauss-Laguerre \\
\hline$R C$ & 11.7270 & 11.7194 & 11.7176 \\
& $(2.602)$ & $(1.975)$ & $(1.992)$ \\
$\theta_{1}$ & 4.8259 & 4.8204 & 4.8191 \\
& $(1.792)$ & $(1.340)$ & $(1.357)$ \\
$\theta_{30}$ & 0.3010 & 0.3010 & 0.3010 \\
& $(0.007)$ & $(0.009)$ & $(0.009)$ \\
$\theta_{31}$ & 0.6884 & 0.6884 & 0.6884 \\
& $(0.008)$ & $(0.009)$ & $(0.009)$ \\
$L$ & -2708.366 & -2708.366 & -2708.366 \\
\hline \hline
\end{tabular}

Table 1: Replication of the original results from Rust (1987) Table IX. 


\begin{tabular}{|c|c|c|c|c|}
\hline & \multicolumn{4}{|c|}{ Bus Groups $1,2,3(N=3,864)$} \\
\hline & Logistic & EBurr3 & EBurr12 & EGB2 \\
\hline \multirow[t]{2}{*}{$R C$} & 11.7176 & 12.1668 & 11.9838 & 12.5549 \\
\hline & $(1.992)$ & $(2.047)$ & $(2.024)$ & $(2.758)$ \\
\hline \multirow[t]{2}{*}{$\theta_{1}$} & 4.8191 & 2.9529 & 4.5258 & 2.6408 \\
\hline & $(1.357)$ & $(1.316)$ & $(1.310)$ & $(1.359)$ \\
\hline \multirow[t]{2}{*}{ Skewness } & 0 & 0.6077 & 0.0747 & 0.6982 \\
\hline & & $(0.272)$ & $(0.104)$ & $(0.279)$ \\
\hline \multirow[t]{2}{*}{ Kurtosis } & 4.2 & 4.3652 & 4.2439 & 4.5422 \\
\hline & & $(0.226)$ & $(0.042)$ & $(0.393)$ \\
\hline Log-likelihood & -2708.366 & -2707.865 & -2708.321 & -2707.851 \\
\hline LR test $p$ value & & 0.3169 & 0.7623 & 0.5971 \\
\hline \multirow[t]{3}{*}{ Hausman test $p$ value } & & 0.8390 & 0.9872 & 0.1506 \\
\hline & \multicolumn{4}{|c|}{ Bus Group $4(N=4,292)$} \\
\hline & Logistic & EBurr3 & EBurr12 & EGB2 \\
\hline \multirow[t]{2}{*}{$R C$} & 10.0732 & 10.1709 & 10.1599 & 10.0567 \\
\hline & $(1.378)$ & $(1.510)$ & $(1.366)$ & $(2.465)$ \\
\hline \multirow[t]{2}{*}{$\theta_{1}$} & 2.2923 & 1.4652 & 2.2107 & 1.5443 \\
\hline & $(0.574)$ & $(1.067)$ & $(0.606)$ & $(1.637)$ \\
\hline \multirow[t]{2}{*}{ Skewness } & 0 & 0.3143 & 0.0278 & 0.2844 \\
\hline & & $(0.343)$ & $(0.044)$ & $(0.511)$ \\
\hline \multirow[t]{2}{*}{ Kurtosis } & 4.2 & 4.1631 & 4.2149 & 4.1273 \\
\hline & & $(0.184)$ & $(0.024)$ & $(0.631)$ \\
\hline Log-likelihood & -3304.155 & -3304.054 & -3304.145 & -3304.048 \\
\hline LR test $p$ value & & 0.6530 & 0.8900 & 0.8988 \\
\hline \multirow[t]{3}{*}{ Hausman test $p$ value } & & 0.7744 & 1.0000 & 0.5100 \\
\hline & \multicolumn{4}{|c|}{ Bus Groups $1,2,3,4(N=8,156)$} \\
\hline & Logistic & EBurr3 & EBurr12 & EGB2 \\
\hline \multirow[t]{2}{*}{$R C$} & 9.7541 & 10.0752 & 9.9865 & 10.2604 \\
\hline & $(0.776)$ & $(0.909)$ & $(0.788)$ & $(1.103)$ \\
\hline \multirow[t]{2}{*}{$\theta_{1}$} & 2.6266 & 0.9671 & 2.3790 & 0.8456 \\
\hline & $(0.488)$ & $(0.530)$ & $(0.494)$ & $(0.607)$ \\
\hline \multirow[t]{2}{*}{ Skewness } & 0 & 0.6653 & 0.0761 & 0.7166 \\
\hline & & $(0.130)$ & $(0.022)$ & $(0.202)$ \\
\hline \multirow[t]{2}{*}{ Kurtosis } & 4.2 & 4.4341 & 4.2448 & 4.5361 \\
\hline & & $(0.158)$ & $(0.014)$ & $(0.355)$ \\
\hline Log-likelihood & -6055.250 & -6053.638 & -6055.099 & -6053.629 \\
\hline LR test $p$ value & & 0.0725 & 0.5818 & 0.1975 \\
\hline Hausman test $p$ value & & 0.9693 & 0.9995 & 0.6799 \\
\hline
\end{tabular}

Table 2: Estimation with flexible distributions. 


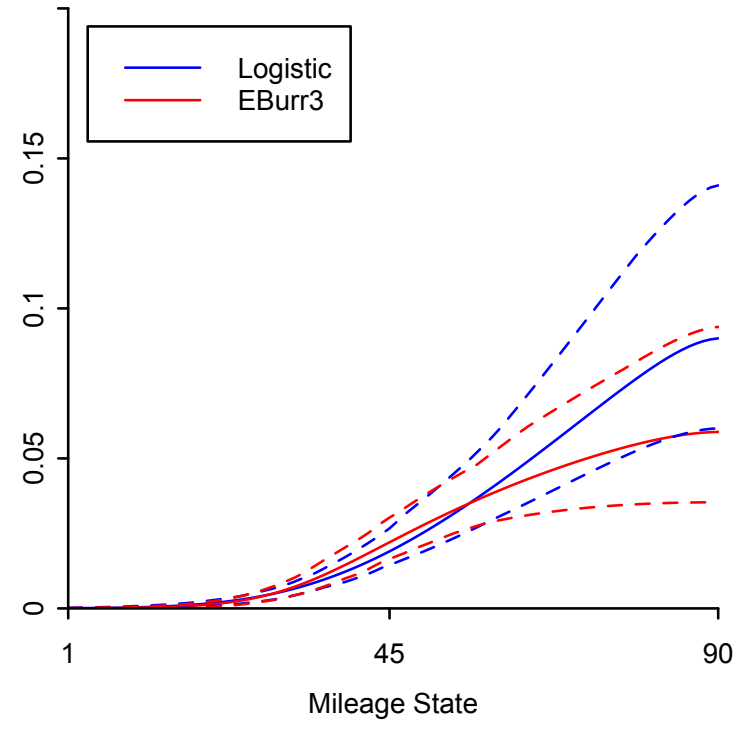

(a) Absolute probabilities.

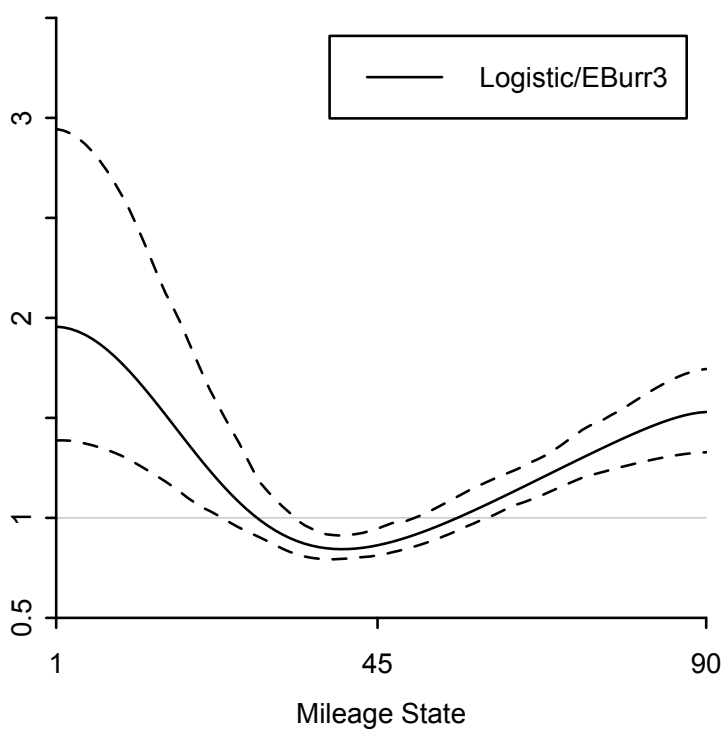

(b) Relative probabilities.

Figure 1: Conditional choice probabilities of engine replacement at each mileage state under the logistic vs. EBurr3 distribution (Bus groups 1-4). 